\title{
Recherches sur des généralisations d'une fonction de Legendre et d'Abel.
}

\author{
(Par Niels Nielsen, à Copenhague.)
}

§ 1. Formules gémḱralus. Points critioues de $L_{n, p}(x)$.

I lest bien connu que Legendre (*), Aber $\left(^{* *}\right)$, Schabrters $\left(^{* * *}\right)$, et dans les dernières années, M. W. KAPTEYN ${ }^{* * * *}$ ) ont démontré pour cette fonction transcendante

$$
L_{2}(x)=\frac{x}{1^{2}}+\frac{x^{2}}{2^{2}}+\frac{x^{3}}{3^{2}}+\cdots+\frac{x^{n}}{n^{2}}+\cdots, \quad|x| \leq 1
$$

un nombre de propriétés remarquables. Dans la communication que voici je me suis proposé d'étudier certaines généralisations de cette fonction célèbre $L_{2}(x)$, de démontrer que les fonctions en question sont holomorphes dans toute l'étendue du plan des $x$, à l'exception des deux points critiques transcendants $x=1$ et $x=\infty$, de plus j'ai à indiquer les branches différentes des ronctions susdites et de donner enfin un nombre de propriétés intéressantes des valeurs obtenues de nos fonctions en $y$ posant $x==1, x=-1$ et $x=\frac{1}{2}$.

Pour construir la généralisation en question de $L_{2}(x)$ définissons comme ordinairement, a l'aide de cette identité,

$$
x(x+1) \ldots(x+n-1)=C_{n}^{0} x^{n}+C_{n}^{1} x^{n-1}+C_{n}^{2} x^{n-2}+\cdots+C_{n}^{n-1} x
$$

les coefficients de la factorielle du rang $n$, puis posons

$$
\omega_{n-1}^{n-p-1}=\frac{C_{n}^{p}}{(n-1) !},
$$

(*) Exercices de calcul integral, t. II, p. 244.

(**) Guvres complettes, t. II, p, 189.

(***) Journol de Crelle, t. 30 .

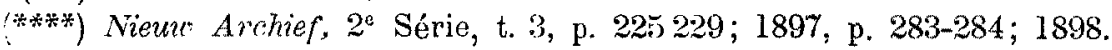


nous aurons partiarlièrement

$$
\omega_{n}^{0}=1, \quad \omega_{n}^{\mathrm{t}}=\frac{1}{1}+\frac{1}{2}+\cdots+\frac{1}{n}, \quad \omega_{n}^{n}=\frac{1}{n !},
$$

tandis que généralement $\omega_{i k}^{p}$ désigne la somme de tous les $\left(\begin{array}{l}n \\ p\end{array}\right)$ produits à $p$ facteurs différents choisis parmi ces $n$ nombres

$$
\frac{1}{1}, \frac{1}{2}, \frac{1}{3}, \ldots, \frac{1}{n},
$$

nous aurons pour le coefficient du binome cette expression

$$
\left(\begin{array}{c}
x+n-1 \\
n
\end{array}\right)=\frac{x}{n} \cdot\left(\begin{array}{c}
\omega_{n-1}^{n-1} \cdot x^{n-1}+\omega_{n-1}^{n-2} \cdot x^{n-2}+\cdots+\omega_{n-1}
\end{array}\right)
$$

tandis qu'une formule bien connue (*) s'écrira sous cette forme nouvelle

$$
(\log (1-x))^{p}=(-1)^{p} p ! \cdot \sum_{s=0}^{s=\infty} \frac{\omega_{p+s-1}^{p-1}}{p+s} \cdot x^{p+s}, \quad|x|<1,
$$

où $p$ désigne un positif entier.

Introduisons maintenant cette fonction beaucoup plus générale que $L_{2}(x)$

$$
L_{n, p}(x)=\sum_{s=0}^{s=\infty} \frac{\omega_{p+s-1}^{p-i}}{(p+s)^{n+1}} \cdot x^{p+s}, \quad|x| \leqq 1
$$

nous aurons particulièrement

tandis que nous posons

$$
L_{0, p}(x)=\frac{(-1)^{p}}{p !} \cdot(\log (1-x))^{p}
$$

$$
L_{n}(x)=\sum_{s=1}^{s=\infty} \frac{x^{s}}{s^{n}}
$$

ce qui donnera cette autre formule particulière

$$
L_{n, 1}(x)=L_{n+1}(x)
$$

Cela posé, appliquons cette formule intégrale

$$
\int_{0}^{1} t^{2-1}(\log t)^{r} d t=\frac{(-1)^{r} r !}{q^{r+1}},
$$

(*) Voir par exemple SaHoöмıцcн, Compendium der höheren Analysis, t. II, p. 13. 
où $r$ désigne un entier non negatif, nous aurons, en vertu de (2), cette expression nouvelle pour la fonction $L_{n, p}(x)$

$$
L_{n, p}(x)=\frac{(-1)^{n+p-1}}{p !(n-1) !} \cdot \int_{0}^{1} \frac{(\log (1-t x))^{p}(\log t)^{n-1}}{t} d t, \quad|x|<1,
$$

où le chemin d'intégration est la partie de l'axe des nombres positifs située entre $t=0$ et $t=1$. Or, cette expression intégral que nous venons de donner se transforme par une intégration par parties; nous aurons en effet

$$
L_{n, p}(x)=\frac{(-1)^{n+p-1} \cdot x}{n !(p-1) !} \cdot \int_{0}^{1} \frac{\left(\log (1-t x)^{p-1}(\log t)^{n}\right.}{1-t x} d t
$$

où le chemin d'intégration est toujours la même partie de l'axe des nombres positifs.

Il est évident que la formule (4) nous fournit un simple moyen pour le prolongement analytique de la fonction $L_{n, p}(x)$ qui n'est définie que pour $|x| \leqq 1$. En effet, dans ce cas l'intégrale susdite représente précisément la série de puissances que nous avons prise comme définition de $L_{n, p}(x)$; pour $p \geq 1$ et $|x|>1$ la même intégrale a toujours une valeur finie et déterminée. Démontrons encore que la fonction de $x$ représentée par cette intégrale est analytique aussi.

Or, la verité de cette assertion est une conséquence immédiate de la forme même de l'intégrale susdite. En effet, nous aurons, en vertu de $\left(4^{\text {bis }}\right)$, pour la dérivée de $L_{n, p}(x)$ cette expression

$$
D_{x} L_{n, p}(x)=\frac{1}{x} \cdot L_{n-1, p}(x),
$$

formule qui est certainement valable pour une valeur finie quelconque de $x$, les valeurs positives et plus grandes que ou égales à 1 exceptées, car dans ce eas l'intégrale figurant au second membre de ( $\left.4^{\text {bis }}\right)$ deviendra illusoire. Ap. pliquons maintenant plusieurs fois la formule (5), nous trouvons

$$
D_{x} L_{1, p}(x)=\frac{1}{x} \cdot L_{0, p}(x)=\frac{(-1)^{p}}{p !} \cdot \frac{1}{x} \cdot(\log (1-x))^{p},
$$

ce qui montre clairement que la fonction $L_{n, p}(x)$ est analytique dans toute partie finie du plan des $x$, à l'exception du point $x=1$ qui est certainement point critique de notre fonction parce que ses dérivées d'un ordre fini mais suffisamment grand deviendront infinies pour cette valeur de $x$. 
Avant d'étudier la nature de ce point critique introduisons les séries numériques obtenues de $L_{n, p}(x)$ en y posant $x=1, x=-1, x=\frac{1}{2}$, séries qui sont très remarquables. Posons pour abréger

$$
L_{n, p}(1)=s_{n, p}, \quad L_{n, p}(-1)=\sigma_{n, p}, \quad L_{n, p}\left(\frac{1}{2}\right)=a_{n, p},
$$

nous aurons particulièrement

$$
\begin{aligned}
s_{n, 1} & =s_{n+1}=\frac{1}{1^{n+1}}+\frac{1}{2^{n+1}}+\frac{1}{3^{n+1}}+\cdots, \quad n \geqq 1 \\
-\sigma_{n, 1}=\sigma_{n+1} & =\frac{1}{1^{n+1}}-\frac{1}{2^{n+1}}+\frac{1}{3^{n+1}}-\cdots, \quad n \geqq 0 \\
a_{n, 1}=a_{n+1} & =\frac{1}{1^{n+1}} \cdot \frac{1}{2}+\frac{1}{2^{n+1}} \cdot \frac{1}{2^{2}}+\frac{1}{3^{n+1}} \cdot \frac{1}{2^{3}}+\cdots, \quad n \geqq 0 .
\end{aligned}
$$

La série $s_{0, p}$ est divergente; pour $\sigma_{0, p}$ et $a_{0, p}$ nous obtenons, au contraire, en vertu de (3), ces expressions

$$
\left.\begin{array}{rl}
\left(-1,{ }^{p} \sigma_{0, p}\right. & =a_{0, p}=\frac{1}{p !} \cdot(\log 2)^{p} \\
a_{1} & =\sigma_{1}=\log 2 .
\end{array}\right\}
$$

Posons encore dans ( $\left.4^{\text {bis }}\right) x=1$ et transformons l'intégrale ainsi obtenue en mettant $1-t$ au lieu de $t$, nous trouvons cette égalité

$$
s_{n, p}=s_{p, n} \text {. }
$$

\section{BranChES DIFFÉRENTES DE $I_{n, p}(x)$.}

Pour déterminer maintenant la nature du point critique $x=1$ de $L_{n, p}(x)$, mettons dans (4) $t x$ au lieu de $t$, nous aurons cette autre expression intégrale

$$
L_{n, p}(x)=\frac{(-1)^{n+p-1}}{p !(n-1) !} \cdot \int_{0}^{x} \frac{(\log (1-t))^{p}(\log t-\log x)^{n-1}}{t} d t
$$

où le chemin d'intégration est représenté par la ligne droite $O A$ qui unit 
les deux points $t=0$ et $t=x$. Appliquons ensuite la formule da binome, et introduisons cette fonction nouvelle

$$
M_{n, p}(x)=\frac{(-1)^{n+p-1}}{p !(n-1) !} \cdot \int_{0}^{x} \frac{(\log (1-t))^{p}(\log t)^{n-1}}{t} d t
$$

où le chemin d'intégration est toujours la même ligne droite $O A$, nous aurons, en vertu de (7), cette autre formule

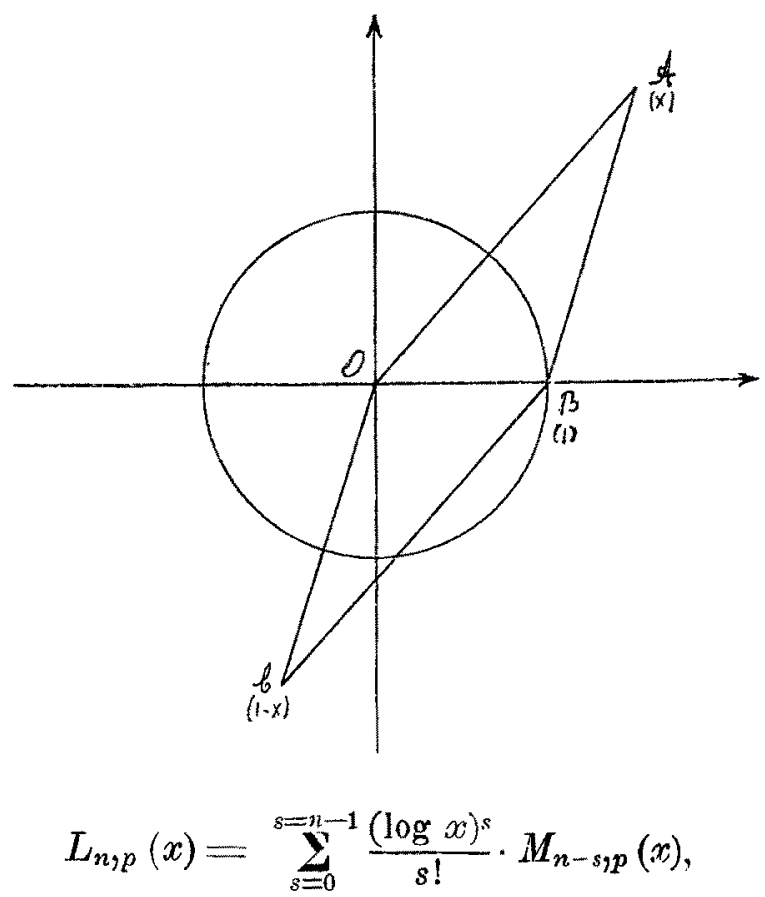

d'où plus particulièrement

$$
\left.\begin{array}{c}
L_{1, p}(x)=M_{i, p}(x) \\
L_{n, p}(1)=M_{n, p}(1)=s_{n, p} .
\end{array}\right)
$$

Inversement, il est très facile d'exprimer la fonction $M_{n, p}(x)$ sous forme d'une fonction linéaire et homogène de

$$
L_{n, p}(x), \quad L_{n-1, p}(x), \quad L_{n-2, p}(x), \ldots L_{1, p}(x) .
$$

En effet, cette expression peut être déduite directement de (8) et des formules analogues qui correspondent aux valeurs plus petites de $n$. Or, cette 
jdentité nous conduira plus facilement au but

$$
\int_{0}^{x} t^{q \cdot 1}(\log t)^{r} d t=\alpha^{q} \cdot \sum_{s=0}^{s} \overline{\overline{\mathbf{z}}} \frac{(-1)^{r} \cdot !}{(r-s) !} \cdot \frac{(\log x)^{r-s}}{q^{s+1}}
$$

nous aurons en effet immédiatement, en vertu de (2),

$$
M_{n, p}(x)=\sum_{s=0}^{s=n-1} \frac{(-1)^{s}(\log x)^{s}}{s !} \cdot L_{n-s, p}(x) \text {, }
$$

formule qui montre clairement que, pour $n>1$, la fonction $M_{n, p}(x)$ est analytique dans toute l'étendue du plan des $x$, à l'exception des points $x=0$, $x=1, x=\infty$ qui sont des points critiques pour cette fonction.

Posons dans (9) $x=\frac{1}{2}$, nous aurons cette formule particulière

$$
M_{n, p}\left(\frac{1}{2}\right)=\sum_{s= \pm 0}^{s=n-1} \frac{\sigma_{t}^{s}}{s !} \cdot a_{n-s, p}
$$

Transformons maintenant par une intégration par parties la définition intégrale de $M_{n, p}(x)$, nous aurons immédiatement

$$
\begin{gathered}
M_{n, p}(x)=\frac{(-1)^{n+p-1}}{n ! p !}(\log x)^{n}(\log (1-x))^{p}+ \\
+\frac{(-1)^{n+p-1}}{n !(p-1) !} \int_{0}^{x} \frac{(\log (1-t))^{p-1}(\log t)^{n}}{1-t} d t
\end{gathered}
$$

d'où, en posant dans la nouvelle intégrale ainsi obtenue $1-t$ au lieu de $t$, cette autre formule

$$
\begin{aligned}
& M_{n, p}(x)=\frac{(-1)^{n+p-1}}{n ! p !} \cdot(\log x)^{n}(\log (1-x))^{p}+ \\
& +\frac{(-1)^{n+p-1}}{n !(p-1) !} \cdot \int_{1-x}^{1} \frac{(\log t)^{p-1}(\log (1-t))^{n}}{t} d t
\end{aligned}
$$

où le chemin d'intégration est la ligne droite $O C$ qui unit les deux points $t=1-x$ et $t=1$. Or, intégrons dans le sens direct le long du périmètre du triangle $O C B$ la fonction

$$
\frac{(\log t)^{p-1}(\log (1-t))^{n}}{t}
$$


nous aurons, en vertu du théorème fondamental de CavcnY

$$
\int_{O C}+\int_{C B}+\int_{B O}=0, \quad \int_{C B}=\int_{O B}-\left.\right|_{O \dot{C}},
$$

de sorte que nous obtenons pour la fonction $M_{n, p}(x)$ cette équation fondamentale

$$
M_{n, p}(x)+M_{p, n}(1-x)=s_{n, p}+\frac{(-1)^{n+p-1}}{n ! p !} \cdot(\log x)^{n}(\log (1-x))^{p},
$$

dont le cas $n=p=1$, qui conduira à $L_{\mathfrak{2}}(x)$, appartient à Legendre.

Posons particulièrement dans (10) $x=\frac{1}{2}$, nous aurons, en vertu de $\left(9^{\text {bis }}\right)$, cette relation numérique

$$
\sum_{r=0}^{r=n-1} \frac{\sigma_{1}^{r}}{r !} \cdot a_{n-r, p}+\sum_{r=0}^{r=p-1} \frac{\sigma_{1}^{r}}{r !} \cdot a_{p-r, n}=s_{n, p}-\frac{\sigma_{1}^{n+p}}{n ! p !},
$$

on bien, en vertu de (6),

$$
\sum_{r=0}^{r} \frac{\sigma_{1}^{n}}{r !} \cdot a_{n-r, p}+\sum_{r=0}^{n} \frac{\sigma_{1}^{n}}{r !} \cdot a_{p-r, n}=s_{n, p}+\frac{\sigma_{1}^{n+p}}{n ! p !}
$$

posons encore dans (11) $p=1$, nous aurons

$$
\sum_{r=0}^{r=n-2} \frac{\sigma_{1}^{n}}{r !} a_{n-r}+a_{i, n-1}=s_{n}-\frac{\sigma_{1}^{n}}{(n-\cdot 1) !}
$$

dont le cas particulier $n=2$, c'est-à-dire la formule

$$
a_{2}=\frac{\pi^{2}}{12}-\frac{1}{2} \cdot(\log 2)^{2}
$$

appartient à LEGENDRE.

Introduisons maintenant dans (8), au lieu des $M_{n, p}(x)$, les expressions tirées de (10), nous obtiendrons, d'après une propriété bien connue des coefficient du binome:

$$
\begin{gathered}
L_{n, p}(x)=\frac{(-1)^{p}}{n ! p !} \cdot(\log c)^{n}(\log (1-x))^{p}+\sum_{r=0}^{r=n-1} \frac{(\log x)^{r}}{r !} \cdot s_{n-r, p}-1 \\
-\sum_{r=0}^{r=n-1} \frac{(\log x)^{r}}{r !} \cdot M_{p, n-r}(1-x)
\end{gathered}
$$


d'où, en vertu de (9), cette formule plus compliquée

$$
\left.\begin{array}{c}
L_{n, p}(x)=\frac{(-1)^{p}}{n ! p !} \cdot(\log x)^{n}(\log (1-x))^{p}+\sum_{r=0}^{r=n-1} \frac{(\log x)^{r}}{r !} \cdot s_{n-r, p}- \\
-\sum_{s=0}^{s=n-1} \frac{(\log x)^{s}}{s !} \cdot \sum_{r=0}^{r=p-1} \frac{(-1)^{r}(\log (1-x))^{r}}{r !} \cdot L_{p-r, n-s}(1-x)
\end{array}\right\}
$$

Considérons en particulier le cas $p=1$, nous obtiendrons cette équation fondamentale plus élégante

$L_{n+1}(x)=\sum_{r=0}^{r=n-2} \frac{(\log x)^{r}}{r !}\left(s_{n-r+1}-L_{i, n \cdots r}(1-x)\right)-\frac{(\log x)^{n}}{n !} \cdot \log (1-x)$,

d'où, en posant $x=\frac{1}{2}$, cette formule numérique

$$
a_{n}=\sum_{r=0}^{r=n-2} \frac{(-1)^{r} \sigma_{1}^{r}}{r !} \cdot\left(s_{n-r}-a_{i, n \cdot r-1}\right)-\frac{(-1)^{n}}{(n--1) !} \cdot \sigma_{1}^{n} \cdot
$$

Or, ces formules générales démontrées, il est très facile de construir toutes les branches diffèrentes des fonctions $L_{n, p}(x)$ et $M_{n, p}(x)$. En effet, désignons pour abréger par $[f(x)]_{a}$ ce qui deviendra $f(x)$ si nous faisons tourner dans le sens direct la variabile $x$ autour du point $x=0$ qui est point critique de $f(x)$, nous aurons immédiatement, à l'ajde de $(9)$ et en appliquant l'identité

$$
[\log x]_{0}=\log x+2 \pi i
$$

cette première équation

$$
\left[M_{n, p}(x)\right]_{0}=\sum_{s=0}^{s=0} \frac{(-1)^{s}}{s !} \cdot(2 \pi i)^{s} \cdot M_{n-s, p}(x),
$$

de sorte que nous obtenons, en vertu de $(10)$, cette autre formule analogue

$$
\left[M_{n, p}(x)\right]_{1}=\sum_{r=0}^{r=1-1} \frac{(-1)^{r}}{r !} \cdot(2 \pi i)\left(M_{p, n-r}(1-x)-s_{n-r, p}\right) .
$$

La formule correspondante pour $\left[L_{n, p}(x)\right]_{1}$ peut être trouvée maintenant à l'aide de (13); cependant elle deviendra assez compliquée, de sorte que nous nous bornerons à déduir de (14) cette formule élégante

$$
\left\lfloor L_{n+1}(x)\right]_{i}=L_{n+1}(x)-\frac{2 \pi i}{n !} \cdot(\log x)^{n}
$$


c'est-à-dire que la ramification de cette fonction est d'un caractère logarithmique.

Après ces remarques générales nous avons à considérer les trois séries numériques $s_{n, p}, \sigma_{n, p}$ et $a_{n, p}$ et à démontrer un nombre de relations remarquables entre ces nombres.

\section{$\S$ 3. SOMMaTION DE LA SÁRIE $s_{n, p}$ A L'AIDE DES NOMBRES $s_{r}$.}

La forme même de l'expression intégrale obtenue de (4) pour $\boldsymbol{s}_{n, p}$ nous conduira naturellement à prendre pour point de départ cette intégrale eulérienne de premier espèce

$$
\int_{0}^{1} t^{x-1}(1-t)^{y-1} d t=\frac{\Gamma(x) \Gamma(y)}{\Gamma(x+y)} \quad \Re(x)>0, \quad \Re(y)>0 .
$$

En effet appliquons la même méthode que dans ma Note précédente, la formule susdite se présente sous cette forme nouvelle

$$
\int_{0}^{1} t^{x-1}(1-t)^{y-1} d t=e^{\gamma(x)+\gamma(y)-\gamma(x+y)}
$$

de sorte que nous obtenons par le même procédé, en différentiant $p$ fois par rapport à $y$,

$$
\begin{gathered}
\int_{0}^{1} t^{x-1}(1-t)^{y-1}(\log (1-t))^{p} d t= \\
=(-1)^{p} p ! e^{\gamma(x)+\gamma(y)-\gamma(x+y)} \cdot \sum_{k=1}^{k=p} \frac{1}{k !} \cdot V(x, y),
\end{gathered}
$$

où nous avons posé pour abréger

et

$$
V^{k, p}(x, y)=\sum \frac{u_{r_{1}}(x, y) u_{r_{2}}(x, y) \ldots u_{r_{k}}(x, y)}{r_{1} \cdot r_{2} . r_{3} \ldots r_{k}}
$$

$$
u_{r}(x, y)=s_{r}(y)-s_{r}(x+y) .
$$

Mettons maintenant dans ces expressions $y=1$, et posons simplement 
$V(x)$ et $u_{r}(x)$ au lieu de $V^{k, p}(x, 1)$ et $u_{r}(x, 1)$ respectivement, nous aurons cette formule plus particulière

$$
\int_{0}^{1} t^{x-1}(\log (1-t))^{p} d t=(-1) p p ! \sum_{k=1}^{k} \frac{1}{k !} \cdot \frac{V^{k, p}(x)}{x},
$$

formule que nous avons à différentier ensuite $(n-1)$ fois par rapport à $x$. Ces calculs faits, mettons $:=0$ et remarquons que nous aurons pour la fonction

cette identité

$$
\varphi(x)=a_{1} x+a_{2} x^{2}+a_{3} x^{3}+\cdots
$$

$$
D_{x}^{n-1}\left(\frac{\varphi(x)}{x}\right)_{x=0}=\frac{1}{n} \cdot \varphi^{(n)}(0)=(n-1) ! a_{n},
$$

nous trouverons finalement, en vertu de (4), pour $x=0$, cette formule générale

$$
s_{n, p}=\frac{(-1)^{n-1}}{n !} \cdot \sum_{k=1}^{n=1} \frac{1}{k !} \cdot\left(D_{x}^{n} V^{k, p}(x)\right)_{x=0} .
$$

Pour effectuer les différentations nécessaires il est bon de se rappeler à cette identité

$$
\begin{gathered}
u_{r}(x)=s_{r}-s_{r}(1+x)=\left(\begin{array}{l}
r \\
1
\end{array}\right) s_{r+1} \cdot x-\left(\begin{array}{c}
r+1 \\
2
\end{array}\right) s_{r+2} \cdot x^{2}+ \\
+\left(\begin{array}{c}
r+2 \\
3
\end{array}\right) s_{r+3} \cdot x^{3}-\cdots
\end{gathered}
$$

Il est evident que la formule (17) nous permet d'exprimer sous forme finie, à l'aide des nombres $s_{r}$, la somme de notre série numérique $s_{n, p}$. En effet, la formule susdite donnera ce théorème général:

La somme de la série numérique $s_{n, p}$ peut s'exprimer sous forme d'un polynome entier des nombres $s_{2}, s_{3}, \ldots s_{n+p}$ et homogène du dégré $(n+p)$ dans ces quantités si nous supposons $s_{r}$ du dégré $r$ les coefficients du polynome susdit sont des nombres rationnels.

Considérer maintenant quelques cas particuliers de ce théorème général. unique

$1 .^{\circ} p=1$; nous n'avons dans ce cas qu'à considérer cette fonction

$$
V^{1,1}(x)=u_{1}(x)
$$

nous retrouvons l'identité $s_{n+1}=s_{n, 1}$. 
$2 .^{\circ} p=2$; ici nous avons à étudier ces deux fonctions

ce qui donnera

$$
\stackrel{1,2}{V^{\prime}}(x)=\frac{1}{2} \cdot u_{2}(x) ; \quad \stackrel{2.2}{V(x)}=\left(u_{1}(x)\right)^{2}
$$

$$
s_{n, 2}=\frac{n+1}{2} \cdot s_{n+2}-\frac{1}{2}\left(s_{2} s_{n}+s_{3} s_{n-1}+\cdots,+s_{n} \dot{s}_{2}\right),
$$

de façon que nous obtiendrons, en posant $(n-1)$ all lieu de $n$ :

$$
\sum_{r=2}^{n} \frac{1}{r^{n t}}\left(\frac{1}{1}+\frac{1}{2}+\cdots+\frac{1}{r-1}\right)=\frac{n}{2} \cdot s_{n+1}-\frac{1}{2} \cdot \sum_{r=1}^{r=1} s_{r} \cdot s_{n r+1}
$$

formule que j'ai démontrée récemment $\left({ }^{*}\right)$ d'une autre manière.

3. $p=3$; nous trouvons ici

$$
V^{1,3}(x)=\frac{1}{3} \cdot u_{3}(x), \quad V^{2,3}(x)=u_{1}(x) u_{2}(x), \quad V^{3,3}(x)=\left(u_{1}(x)\right)^{3},
$$

ce qui donnera

$$
s_{n, 3}=\frac{(n+2)(n+1)}{6} s_{n+3}-\frac{1}{2} \cdot \sum_{r=0}^{r=n-1}(n-r) s_{r+2} \cdot s_{n-r+1}+\frac{1}{6} \cdot \sum_{r=0}^{r=n-3} s_{r+2-2} \cdot \alpha_{n-r+1},
$$

où nous avons posé pour abréger

$$
o_{p}=s_{2} s_{p-2}+s_{3} s_{p_{3}}+\cdots+s_{p-2} s_{2} .
$$

Dans le cas $n=7$ nous trouvons par exemple

$$
\begin{aligned}
s_{7,3}= & 12 s_{40}-\left(\frac{7}{2} s_{8} s_{2}+4 s_{7} s_{3}+4 s_{6} s_{4}+1 s_{5}^{2}\right)+ \\
& +\left(\frac{1}{2} s_{6} s_{2}^{2}+\frac{1}{3} s_{1}^{2} s_{2}+\frac{1}{2} s_{3}^{2} s_{4}+s_{2} s_{3} s_{5}\right) .
\end{aligned}
$$

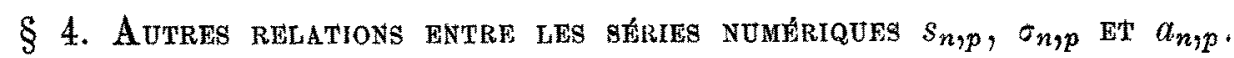

Les formules générales que nous venons de développer au $\S 2$ nous ont donné comme des cas particuliers quelques formules contenant les séries $a_{n, p}$, tandis que les mêmes formules générales ne nous donnent aucun moyen pour

(*) Ce Journal, même volume, p. 195.

Annali di Matematica, Serie III, tomo IX. 
introduire directement les séries analogues $\sigma_{n, p}$. Or, la formule $\left(g^{\text {bis }}\right)$ donne si nous posons respectivement

$$
x=1, \quad t=\sin ^{2} \varphi ; \quad x=-1, \quad t=\operatorname{tg}^{2} \varphi ; \quad x=\frac{1}{2}, \quad t=2 \sin ^{2} \varphi
$$

ces expressions intégrales pour nos trois séries numériques

$$
\begin{gathered}
s_{n, p}=\frac{(-1)^{n+p-1} \cdot 2^{n+p}}{n !(p-1) !} \cdot \int_{0}^{\frac{\pi}{2}} \operatorname{tg} \varphi \cdot(\log \cos \varphi)^{p-1}(\log \sin \varphi)^{n} d \varphi \\
\sigma_{n, p}=\frac{(-1)^{n-1} \cdot 2^{n+p}}{n !(p-1) !} \cdot \int_{0}^{\frac{\pi}{4}} \operatorname{tg} \varphi \cdot(\log \cos \varphi)^{p-1}(\log \operatorname{tg} \varphi)^{n} d \varphi \\
a_{n, p}=\frac{(-1)^{n+p-1} \cdot 2 p}{n !(p-1) !} \cdot \int_{0}^{\frac{\pi}{4}} \operatorname{tg} \varphi(\log \cos \varphi)^{p-1}\left(\log \left(2 \sin ^{2} \varphi\right)\right)^{n} d \varphi,
\end{gathered}
$$

formules qui nous permettent de déduire facilement un nombre de formules nouvelles entre nos trois séries numériques.

Posons dans (18) (19) (20) $p=1$, nous trouvons des expressions intégrales pour $s_{n+1}, \sigma_{n+1}$ et $a_{n+1}$.

Considérons maintenant en premier lieu cette intégrale définie:

$$
A_{n, p}=\frac{(-1)^{n+p-1} \cdot 2^{n+-p}}{n !(p-1) !} \cdot \int_{0}^{\frac{\pi}{4}} \operatorname{tg} \varphi(\log \cos \varphi)^{p-1}(\log \sin \varphi)^{n} d \varphi,
$$

nous aurons, en posant

$$
\log \sin \varphi=\log \operatorname{tg} \varphi+\log \cos \varphi
$$

et en appliquent la formule du binome, à l'aide de (19), pour $A_{n \cdot p}$ cette première expression

$$
A_{n, p}=(-1)^{p} \cdot \sum_{r=0}^{r=n}(-1)^{\nu}\left(\begin{array}{c}
p+r-1 \\
r
\end{array}\right) \cdot \sigma_{n-r, p+r} .
$$

Posons ensuite dans $\S 2,(\beta) x=\frac{1}{2}, t=\cos ^{2} \varphi$, nous aurons

$$
M_{n, p}\left(\frac{1}{2}\right)=-\frac{\sigma_{1}^{n+p}}{n ! p !}+A_{n, p}
$$


ce qui donnera, en vertu de $\left(9^{\text {bis }}\right)$ et de $(6)$, pour $A_{n, p}$ cette autre expression

$$
A_{n, p}={ }_{r=0}^{r=\bar{\Sigma}^{n}} \frac{\sigma_{1}^{r}}{r !} \cdot a_{n-r, p}
$$

d'où, en vertu de (21),

$$
\sum_{r=0}^{r=n^{n}} \frac{\sigma_{1}^{r}}{r !} \cdot a_{n-r \cdot p}=(-1)^{p} \sum_{r=0}^{r=\sum^{n}}(-1)^{r}\left(\begin{array}{c}
p+r-1 \\
r
\end{array}\right) \cdot \sigma_{n-r, p+r}
$$

meitons particulièrement $n=1$, nous aurons par là

$$
a_{s, p}=(-1)^{p} \sigma_{1, p}-\frac{\sigma_{1}^{p+1}}{(p+1) !} .
$$

Introduisons encore dans (10) au lieu de $M_{n, p}\left(\frac{1}{2}\right)$ l'expression (a), nous aurons

$$
A_{n, p}+A_{p, n}=s_{n, p}+\frac{\sigma_{1}^{n+p}}{n ! p !},
$$

d'où, en vertu de (21) et (6), cette autre formule

$$
\begin{gathered}
(-1)^{p} \sum_{r=0}^{r=n-1}(-1)^{r}\left(\begin{array}{c}
p+r-1 \\
r
\end{array}\right) \cdot \sigma_{n-r, p+r}+ \\
+(-1)^{n} \sum_{r=0}^{r=p-1}(-1)^{r}\left(\begin{array}{c}
n+r-1 \\
r
\end{array}\right) \cdot \sigma_{p-r, n+r}=s_{n, p},
\end{gathered}
$$

ce qui donnera pour $p=1$ et $n-1$ au lieu de $n$, ce cas particulier très élégant

$$
\sum_{r=1}^{c=n-2}(-1)^{r} \sigma_{n-r, r}=s_{n}+(-1)^{n} \cdot 2 \cdot \sigma_{i, n-1} .
$$

Posons ensuite dans (19)

et dans (20)

$$
\log \operatorname{tg} \varphi=\log \sin \varphi-\log \cos \varphi
$$

$$
\log \left(2 \sin ^{2} \varphi\right)=\sigma_{1}+2 \log \sin \varphi,
$$

la formule du binome donnera, en vertu de la définition de $A_{n, p}$,

$$
\begin{gathered}
\sigma_{n, p}=(-1)^{p} \sum_{r=0}^{r-n}(-1)^{r}\left(\begin{array}{c}
p+r-1 \\
r
\end{array}\right) A_{n-r, p+r} \\
a_{n, p}=\sum_{r=0}^{r=} \frac{(-1)^{r}}{r !} \cdot \sigma_{3}^{r}, A_{n-r, p+r} .
\end{gathered}
$$


Cela posé, exprimons dans (24) tous les nombres $A_{r, s}$ à l'aide de $\left(21^{\text {bis }}\right)$, nous trouverons cette proposition remarquable:

La somme de la série numérique $\sigma_{n, p}$ s'exprine sous forme d'une fonction linéaire et homogène des séries $a_{r, s}$ pour lesquelles $r \leqq n, s \geqq p$; les coefficients de cette expression sont des polynomes entiers et à coefficients rationnels de $\sigma_{1}=\log 2$. De plus, supposons $a_{r, s} d u$ dégré $(r+s)$ et $\sigma_{1}$ du dégré 1, l'expression susdite est homogène du dégré $(n+p)$.

Exprimons, au contraire, dans $\left(24^{\text {bis }}\right)$ tous le nombres $A_{, y_{s}} \grave{a}$ l'aide de (21), nous verrons que le théorème que nous venons, d'indiquer restera rrai encore si nous y permutons les séries $a_{r, s}$ et $\sigma_{r, s}$.

Ces théorèmes démontrés, il est évident que pour connaître toutes les

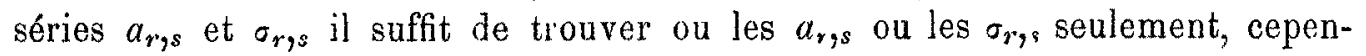
dant je n'ai pas réussi à résoudre ce problème.

Pour établir quelques autres relations entre les séries $s_{n, p}$ et $\sigma_{n, p}$ considérons encore cette intégrale définie

$$
J_{n}=\frac{(-1)^{n-1}}{(n-1) !} \cdot \int_{0}^{\frac{\pi}{4}} \operatorname{tg} \varphi(\log \cos \varphi+\log \sin \varphi)^{n+1} d \varphi,
$$

nous aurons de (19), en posant

$$
\log \cos \varphi+\log \sin \varphi=\log \operatorname{tg} \varphi+2 \log \cos \varphi
$$

et en appliquant ensuite la formule du binome, pour $J_{n}$ cette première expression

$$
J_{n}=\frac{1}{2^{n}} \cdot \sum_{r=0}^{r=1 n-1}(-1)^{r+1} \cdot 2^{r+1} \cdot \sigma_{n-r-1, r+1} \cdot
$$

Introduisons ensuite dans $(\beta)$ pour $A_{n, p}$ et $A_{p, n}$ les expressions intégrales correspondantes, et posons $r$ au lieu de $p$ et $n-r$ au lieu de $n$, nous aurons

$$
\begin{aligned}
& \left(\begin{array}{l}
n-1 \\
r-1
\end{array}\right) \int_{0}^{\frac{\pi x}{4}} \operatorname{tg} \varphi(\log \cos \varphi)^{r-1}(\log \sin \varphi)^{n-r} d \varphi+ \\
& \left.+\left(\begin{array}{c}
n-r-1 \\
n-1
\end{array}\right) \int_{0}^{\frac{x}{4}} \operatorname{tg} \varphi(\log \cos \varphi)^{n-r-1} \log \sin \varphi\right) d \varphi= \\
& =\frac{(-1)^{n-1}}{2^{n} \cdot n} \cdot\left(\begin{array}{l}
n \\
r
\end{array}\right) \cdot \sigma_{1}^{n}-\frac{(-1)^{n}(n-1) !}{2^{n}} \cdot s_{n-r, r}
\end{aligned}
$$


Posons maintenant dans cette formule $r=1, r=2, r=3, \ldots$, $r=n-1$, puis ajoutons toutes ces équations, nous aurons à l'aide de la formule de binome, et après un simple calcul, pour $J_{n}$ cette autre expression

$$
J_{n}=\frac{1}{2} \cdot \frac{\sigma_{1}^{n}}{n !}+\frac{1}{2^{n+1}} \cdot 2_{1}^{n-1} s_{n-r, r},
$$

ce qui donnera, en vortu de $(25)$ cette nouvelle formule numérique

$$
\sum_{1}^{n-1} s_{n-r, r}=\sum_{1}^{n-1}(-1)^{r} 2^{r} . \sigma_{n-r, r}
$$

Mettons encore dans $(\gamma)(2 n-1)$ au lieu de $n$ et successivement $r=1$, $2,3, \ldots, 2 n-1$, puis ajoutons avec des signes alternés toutes ces équations, la formule du binome donnera, après un simple calcul et à l'aide de (19) pour $p=1$, cette autre formule numérique

$$
2 \sigma_{2 n}=\sum_{1}^{2 n-1}(--1)^{r-1} \cdot s_{2 n-r, r}
$$

ou bien, à l'aide de l'identité $s_{n, p}=s_{p, n}$

$$
\sigma_{2 n}=\sum_{1}^{n-1}(--1)^{r-1} s_{2 n}, r,-\frac{(-1)^{n}}{2} \cdot s_{n, n} .
$$

Il est digne d'être remarqué que cette formule numérique n'est au fond autre chose que cette formule intégrale bien connue

$$
\int_{0}^{1} t^{x-1}(1-t)^{-x} d t=\frac{\pi}{\sin \pi x}, \quad 1>\Re(x)>0
$$

ou, en appliquant la formule du binome et en intégrant terme à terme la série infinie ainsi obtenue, ce qui est permis:

$$
\frac{\pi}{\sin \pi x}=\frac{1}{x}+\sum_{s=1}^{s=\infty} \frac{x(x+1) \ldots(x+s-1)}{1.2 .3 \ldots 3} \cdot \frac{1}{x+s} \quad \Re(x)<1 .
$$

Cherchons en effet aux deux membres de cette formule le coefficient de $x^{2 n}$, nous trouvons précisément, en vertu de (1), la relation (27).

En terminant ces recherches nous avons encore à indiquer un autre groupe de formules qui contiennent les séries $s_{n, p}$ et $\sigma_{n, p}$. A cet égard prenons comme point de départ cette formule bien comme

$$
\int_{0}^{\infty} t^{x-1}(1+t)^{-y} d t=\frac{\Gamma(x) \Gamma(y-x)}{\Gamma(y)}, \quad \Re(x)>0, \quad \Re(y-x)>0,
$$


puis mettons

$$
\int_{0}^{\infty}=\int_{0}^{1}+\int_{1}^{\infty}
$$

et posons dans la dernière des intégrales nouvelles $\frac{1}{t}$ au lieu de $t$, nous aurons

$$
\int_{0}^{1} t^{x-1}(1+t)^{-y} d t+\int_{0}^{1} t y-\infty-1(1+t)^{-y} d t=e^{\gamma(x)+\gamma(y-x)-\gamma(y)} .
$$

Différentions maintenant $p$ fois par rapport à $y$ cette dernière formule, puis posons $y=1$, le procédé ordinaire donnera sans peine

$$
\begin{gathered}
\frac{1}{p !} \cdot \int_{0}^{1} \frac{t^{x-1}\left(\left.\log (1+t)\right|^{p}\right.}{1+t} d t+ \\
+\sum_{s=0}^{s=p} \frac{(-1)^{s}}{s !(p-s) !} \cdot \int_{0}^{1} \frac{t^{-x}(\log t)^{s}(\log (1+t) p-s)}{1+t} d t= \\
=\frac{\pi x}{\sin \pi x} \cdot \sum_{k=1}^{k=p} \frac{(-1)^{k}}{k !} \cdot \frac{V(-x)}{x}
\end{gathered}
$$

où $V^{k . p}(x)$ est la même fonction que dans la formule (17).

Cela posé, mettons pour abréger

$$
B_{r, p}=\sum_{k=1}^{h=p} \frac{(-1)^{k}}{k !} \cdot D_{x}^{r}\left(\frac{V(-x)}{x}\right)_{x=0},
$$

puis différentions $(n-1)$ fois par rapport à $x$ la formule $(\delta)$, nous aurons finalement, en posant ensuite $x=0$ et en appliquant les deux expressions intégrales obtenues pour $\sigma_{n, p}$ en mettant dans (4) et $\left(4^{\text {bis }}\right) x=-1$, cette formule nouvelle

$$
\left.\begin{array}{c}
\sigma_{n, p}+\sigma_{n-1, p+1}+(-1)^{n} \cdot \sum_{r=0}^{r=0}(-1)^{r}\left(\begin{array}{c}
n+r-1 \\
r
\end{array}\right) \sigma_{n+r-1, p-r+2}= \\
=(-1)^{n+p-1} \cdot \sum_{r=0}^{\sum^{2}} \frac{2 \sigma_{2 r}}{(n-2 r) !} \cdot B_{n-2 r, p},
\end{array}\right\}
$$

où nous avons posé pour abréger $2 \sigma_{0}=1$. 
La formule générale (28) est très compliquée, il est vrai, cependant il est très facile de déduire de cette formule plusieurs autres que nous venons de démontrer. Indiquons quelques-uns de ces cas particuliers.

$1 .^{0} n=1$; nous retrouvons la formule $\left(23^{\text {bis }}\right)$.

$2 .^{\circ} p=1$; nous aurons dans ce cas

$$
B_{r, 1}=-\left(D_{r}^{r} V^{1,1}(-x)\right)_{x=0},
$$

d'où, en vertu de $\left(17^{\text {bis }}\right)$,

$$
B_{r, 1}=r ! s_{r+2}
$$

ce qui donnera cette formule particulière

$$
-\left(n-(-1)^{n}\right) \sigma_{n, 4}+2 \cos ^{2} \frac{n \pi}{2} \cdot \sigma_{n-1,2}=s_{n+1}+2 \sum_{r=1}^{\leq \frac{n-1}{2}} \sigma_{2 r} . s_{2 n-2 r+1},
$$

d'où, en posant $(2 n-1)$ au lieu de $n$, cette formule récursive

$$
\sum_{1}^{n-1} \sigma_{2 r} s_{2 n-2 r}=n \sigma_{2 n}-\frac{1}{2} \cdot s_{2 n},
$$

que j'ai démontrée récemment $\left(^{*}\right)$ d'une autre maniềre; c'est la même chose pour la formule analogue que nous trouvons en posant $2 n$ an lieu de $n$.

Copenhague, le 26 avril 1903.

(*) Ce Journal, même volume, p. 196. 